\title{
The relationship between individual risk and cost-effectiveness in screening interventions*
}

\author{
Christopher James Sampson, MSc [1] $\quad$ Marilyn James, PhD [1] \\ David Whynes, MLitt [2]
}

November 29, 2013

[1] School of Sociology and Social Policy, University of Nottingham

[2] School of Economics, University of Nottingham

\footnotetext{
${ }^{*}$ Financial support for this study was provided in part by a grant from the National Institute for Health Research. The funding agreement ensured the authors' independence in designing the study, interpreting the data, writing, and publishing the report. The views expressed in this publication are those of the authors and not necessarily those of the NHS, the NIHR or the Department of Health.

${ }^{\dagger}$ Correspondence to: C J Sampson, Institute of Mental Health, University of Nottingham Innovation Park, Triumph Road, Nottingham, NG7 2TU, UK. Email: chris.sampson@ nottingham.ac.uk
} 


\begin{abstract}
Advancements in our understanding of the causes and correlates of disease mean that we are now able to estimate an individual's level of risk. This, and the ever-increasing need for healthcare interventions to be cost-effective, has led to calls for the introduction of risk-based screening. Risk-based screening would involve the use of information about an individual's risk factors to decide whether or not they should be eligible for screening, or the frequency with which they should be invited to attend screening. Evidence is emerging that targeted screening, towards those at higher risk, can increase the cost-effectiveness of a screening programme. The relationship between individual risk and the cost-effectiveness of screening an individual is implicitly recognised in current population screening programmes in the UK. However, the nature of this relationship, and its implications for cost-effectiveness analysis, has not been presented in the academic literature. In this study we propose that an individual's risk of developing a disease has a consistent and quantifiable relationship with the cost-effectiveness of screening them. We suggest a simple modification to standard methods of cost-effectiveness analysis that enables the incorporation of individual risk. Using numerical examples we demonstrate the nature of the relationship between risk and cost-effectiveness and suggest means of optimising a screening intervention. This can be done either by defining a minimum level of risk for eligibility or by defining the optimal recall period for screening. We suggest that methods of decision modelling could enable such an analysis to be carried out, and that information on individual risk could be used to optimise the cost-effectiveness of population screening programmes.
\end{abstract}

Keywords: Risk-based screening; Prevention; Decision modelling; Economic evaluation; Personalised medicine

JEL: $\mathrm{I} 10$ 


\section{Introduction}

It is often the case that the earlier a disease is identified, the greater the effect that treatment can have. Screening is a way of identifying those in the early stages of disease, or those at increased risk of disease, in order that they might be given effective treatment or engaged in preventative measures. Screening interventions are somewhat unusual in that they involve a healthcare intervention for an individual who is apparently in good health. By definition, screening identifies those who can and those who cannot benefit from treatment. Screening interventions, in themselves, usually confer little or no benefit. As such, assuming we seek to maximise an individual's health, the extent to which a screening intervention can be effective depends on the effectiveness of treatment or care following a positive screen. If an individual is more likely to screen positive it is more likely that they will receive the intended benefits of screening. Whether or not an individual benefits from screening is therefore dependent on their likelihood of screening positive. Little or no health benefit is to be gained in being screened negative.

An individual's screening outcome is not random; the probability of screening positive is dependent on a set of (observed or unobserved) risk factors. Advancements in our understanding of the causes and correlates of disease, and improvements in routine data collection, mean that it is now possible, in many cases, to estimate an individual's risk of developing a disease within a given period of time. Given the simple assertions outlined thus far we can infer that it is more likely to be cost-effective to screen an individual at high risk of disease than to screen an individual at low risk of disease. It is for these reasons that more individualised screening programmes are currently being proposed by academics and decision-makers alike. Such screening programmes, which would consider individual risk in their design, will have an important question to answer: at what level of risk should an individual be referred to screening? In some cases, where repeated screening is necessary, programmes will also need to differentiate screening intervals based on individual risk. Currently there is no generally accepted way of defining a threshold level of risk for screening eligibility.

This study presents one possible way of defining a threshold level of risk; based on cost-effectiveness. The framework could also be adapted to consider other maxi- 
mands. We propose that there is a consistent and quantifiable relationship between the cost-effectiveness of a preventative intervention, such as screening, and an individual's estimated level of risk. The framework could be used to inform the design of risk-based screening programmes, which may be implemented in the future. Section 2 of this paper reviews the background to risk-based screening. Section 3 defines the relationship between individual risk and the cost-effectiveness of screening, employing numerical examples and demonstrating the potential for optimisation. Section 4 discusses the ethical and policy implications of the approach and section 5 concludes.

\section{Background}

\subsection{Screening}

Screening involves the attempted identification of asymptomatic disease, for which early intervention could prevent irreversible effects of disease progression. Effective screening interventions can reduce the long-term burden of disease in many clinical areas and can potentially be cost-saving. As with all healthcare interventions, it is important that screening programmes be cost-effective; they must consider cost of provision; the expected benefits; and any potential for harm. Designing screening programmes appropriately - knowing who to screen, how to screen them and how often is a difficult undertaking.

In the UK, the National Screening Committee (NSC) was founded to develop a framework for population screening programmes provided by the National Health Service (NHS). A number of population screening programmes have since been developed. Most national screening programmes in England share a number of characteristics. Most involve a one-off screening intervention or series of interventions, and no intervallic recurrence. The majority have no explicit aim besides the early detection and treatment of disease, with the goal of increasing the effectiveness of treatment and reducing the burden of disease. All are limited to a particular subset of the population, and most are aimed at either pregnant women or newborn babies. Those programmes designed for adults, such as cancer and abdominal aortic aneurysm screening 
programmes, tend to have a minimum age of eligibility.

One way of ensuring that screening programmes represent good value is to differentiate them based on individuals' characteristics. At present, eligibility for screening in publicly-provided programmes is usually defined by a lead risk factor, such as age, sex or presence of a related disease. It is unusual for publicly-provided screening programmes to be available to all populations. The nature of screening tends to be homogeneous for all participants, regardless of their differentiating characteristics. The policy of screening based on people's risk seeks to alter this. At its most basic level it involves defining groups of high risk and low risk individuals and deciding their eligibility, or offering them different interventions, accordingly. The purpose of this is to increase the overall cost-effectiveness of screening by reducing the ratio of true negative to true positive screens, potentially with a reduced overall cost. While such programmes remain unadopted, it is possible that they will be more widespread in the future.

\subsection{Risk}

We use the word risk in the sense of an individual's hazard rate or hazard function. That is, an individual with a given set of characteristics, at any moment in time, can be ascribed a hazard rate representing the probability that an event will occur. Mathematically this is derived as the limit of a number of events occurring in a given unit of time, divided by the number of individuals at risk over time. In order to estimate an individual's hazard rate we use information collected from relevant hazard ratios, the effect of which is estimated by treating the log of the hazard rate as a function of baseline hazard and explanatory variables. Hazard ratios represent the effect on an individual's hazard rate of various characteristics; i.e. risk factors. These are calculated using survival analysis or time-to-event analysis, which involves the use of proportional hazard models informed by existing data and by expert opinion. Such models are able to estimate the probability that an individual will develop a given disease within a given period of time within a given margin of error. 


\subsection{Risk-based screening}

Current screening programmes implicitly consider risk by offering screens to a limited section of the population. Age is a risk factor common across most areas of health and disease. Similarly, men and women tend to have different risk levels for disease, and some screening programmes are differentiated by sex, or offered to only men or only women. It is clear that risk is a consideration in the design of NSC screening programmes in the UK. Current programmes tend to consider only one or two risk factors when differentiating screening for different individuals. These are usually general characteristics such as age or sex. A number of cost-effectiveness analyses of screening interventions that take account of individuals' risk factors have been carried out. Using a Markov model to evaluate colorectal cancer screening, Dan et al find that selective screening, based primarily on an individual's age, is more effective than standardised screening[1]. Similarly, Lansdorp-Vogelaar et al find a small benefit associated with individualised colonoscopy screening [2]. Also using decision modelling methods, Round et al find that the most cost-effective screening method for gestational diabetes mellitus depends on a woman's individual risk of disease[3]. Similarly, Aus et al find that screening intervals for prostate cancer should be individualised based on prostate-specific antigen levels[4].

Recently there have been calls for individualised screening [5] and assertions that, in the future, a greater emphasis must be placed on risk-based screening [6]. In the UK, proposals are in place to implement what we describe here as 'risk-based screening'; a screening programme based upon an explicitly estimated level of individual risk, calculated using data on all known risk factors [7, 8]. The likely outcome of such studies is that a finite number of risk categories will be defined and screening will be differentiated depending on into which category an individual falls. However, in the extreme, screening programmes could be individualised on a per-person basis and could be very specifically tailored to the individual.

One clinical area where such a programme is being trialled is in screening for diabetic retinopathy [8]. In their attempt to optimise screening intervals for diabetic retinopathy, Aspelund et al develop a model based on a fixed number of diagnoses 
across screening intervals[9]. Their model suggests that optimal screening intervals range from 6 to 60 months, depending on an individual's level of risk. Mehlsen et al adopt a similar method, using a fixed risk margin of $0.5 \%$ chance of event [10]. They use multiple logistic regression to find the optimal screening interval for low-risk diabetic retinopathy patients, and find that screening intervals should be extended for most low-risk individuals. Similarly, Chalk et al find that extending the screening interval for low-risk individuals is a cost-effective strategy[11]. Davies et al [12] suggest that simulation methods could be used to stratify individuals based on their risk level, and that screening intervals could be adjusted accordingly, but the authors believe this to be impractical.

\section{Risk and cost-effectiveness}

A relationship between an individual's level of risk and the cost-effectiveness of screening them has been implicitly acknowledged by policy-makers and research funding bodies. However, the nature of this relationship and the ways in which it might be harnessed have not been investigated. Here we present a stylised example of riskbased screening using data relating to two screening programmes currently operating in the UK; the NHS Diabetic Eye Screening Programme and the NHS Bowel Cancer Screening Programme. The example is designed such that it could apply to any basic cost-effectiveness analysis of a screening programme when compared with no screening. In the case of diabetic retinopathy screening we use reported figures from a cost-effectiveness analysis of systematic photographic screening compared with opportunistic screening [13], though we assume that the study compared systematic screening with no screening for the sake of the demonstration. For bowel cancer we present figures from a trial of screening for colorectal cancer [14, 15]. The primary outcome is cost per true positive screen; an outcome relevant, if not paramount, to all screening programmes. 


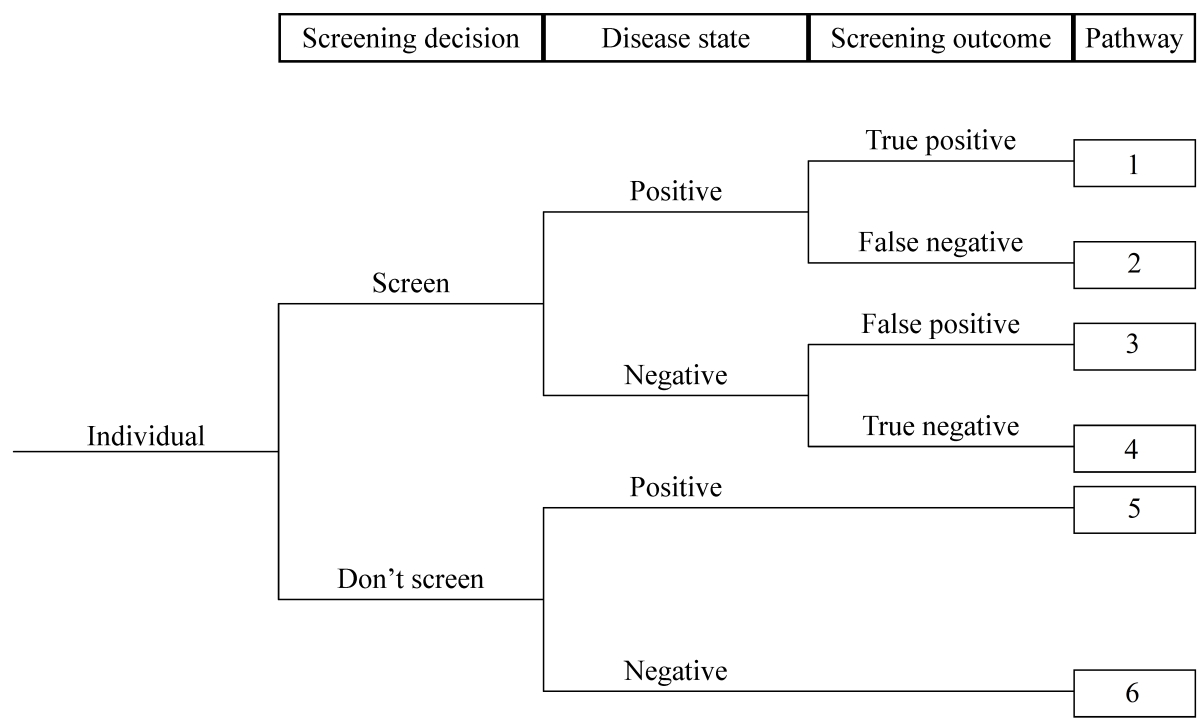

Figure 1: Screening pathways

\subsection{Defining the relationship}

The cost-effectiveness of an intervention is generally presented in the form of an incremental cost-effectiveness ratio (ICER), estimated as:

$$
I C E R=\frac{\left(C_{1}-C_{0}\right)}{\left(E_{1}-E_{0}\right)}
$$

where $C_{1}$ and $E_{1}$ represent the cost and effect of intervention '1' and $C_{0}$ and $E_{0}$ represent the cost and effect of intervention ' 0 '. However, these values are sample means and are the product of a number of possible prognoses and treatment pathways. Therefore, the values in equation 1 are expected values; they are defined by probabilities of various events occurring. In traditional trial-based studies these probabilities may not be observed, while in model-based studies they tend to be explicitly defined. Whether observed or unobserved, cost-effectiveness estimates are partly defined by a set of probabilities.

The expected incremental costs and outcomes of a screening intervention, at the individual level, are dependent on at least two binary probabilities; whether the individual has a given disease and whether they are screened positive or negative. Allowing for 
false positives and false negatives, this means there will be four possible outcomes for an individual who is screened and two for an individual who is not. These six possible pathways are shown in Figure 1

Therefore, for a given population or individual, ICER calculations in the evaluation of screening interventions are dependent upon at least three probabilities; the probability of a positive disease state, the false negative rate and the false positive rate. For the screening intervention in Figure 1 , the probability of each pathway for the individual, depending on whether they are screened or not, is:

$$
\begin{aligned}
& \operatorname{Prob}(1)=r \times(1-\beta) \\
& \operatorname{Prob}(2)=r \times \beta \\
& \operatorname{Prob}(3)=(1-r) \times \alpha \\
& \operatorname{Prob}(4)=(1-r) \times(1-\alpha) \\
& \operatorname{Prob}(5)=r \\
& \operatorname{Prob}(6)=1-r
\end{aligned}
$$

Where $r$ is the probability that the disease (e.g. diabetic eye disease) is present, $\alpha$ is the false positive rate and $\beta$ is the false negative rate of the screening intervention. The probability that an individual has the disease $(r)$ corresponds to the incidence or prevalence of the disease (depending on the nature of the intervention) in the population eligible for screening. In trials, $r$ is only observed at the sample level. In modelling studies, $r$ is usually estimated at the population level. Our primary assertion in this paper is that, given recent developments in the estimation of risk, $r$ can be estimated for an individual. This would be in the form of a hazard rate, as described above.

In practice the figures in equations 277 may not be known, but they are the underlying probabilities in defining an individual's pathway. The expected incremental cost of screening $\left(C_{1}-C_{0}\right)$ and the expected incremental effect $\left(E_{1}-E_{0}\right)$ are dependent on 
these probabilities. As such, $C_{1}, C_{0}, E_{1}$ and $E_{0}$ are defined as:

$$
\begin{aligned}
& C_{1}=\sum_{k=1}^{4}(\operatorname{Prob}(k) \times C(k)) \\
& C_{0}=\sum_{k=5}^{6}(\operatorname{Prob}(k) \times C(k)) \\
& E_{1}=\sum_{k=1}^{4}(\operatorname{Prob}(k) \times E(k)) \\
& E_{0}=\sum_{k=5}^{6}(\operatorname{Prob}(k) \times E(k))
\end{aligned}
$$

Where $k=1,2 \ldots 6$ are the possible pathways shown in Figure 1 Assuming a fixed cost of screening, and zero cost of no screening, we can estimate the expected costs and outcomes of screening, as shown in Table 1

\begin{tabular}{l|l|l|l|l|l} 
Pathway & Probability & Cost & Expected cost & Outcome & Expected outcome \\
\hline Screening & & & & & \\
1 & $r(1-\beta)$ & $C_{S}$ & $(r(1-\beta)) C_{S}$ & $E_{S}$ & $(r(1-\beta)) E_{S}$ \\
2 & $r(\beta)$ & $C_{S}$ & $(r(\beta)) C_{S}$ & 0 & $(r(\beta)) 0$ \\
3 & $(1-r)(\alpha)$ & $C_{S}$ & $((1-r)(\alpha)) C_{S}$ & 0 & $((1-r)(\alpha)) 0$ \\
4 & $(1-r)(1-\alpha)$ & $C_{S}$ & $((1-r)(1-\alpha)) C_{S}$ & 0 & $((1-r)(1-\alpha)) 0$ \\
Total & 1 & & $C_{1}$ & & $E_{1}$ \\
\hline No screening & & & & & $(r)(0)$ \\
5 & $r$ & 0 & $(r) 0$ & 0 & $(1-r)(0)$ \\
6 & $(1-r)$ & 0 & $(1-r) 0$ & 0 & $E_{0}$ \\
Total & 1 & & $C_{0}$ & &
\end{tabular}

Table 1: Expected costs and outcomes

For the purpose of our demonstration we can now explicitly include ' $r$ ' in the calculation of the ICER. This is done by substituting the new expressions for probabilityweighted costs and outcomes, shown in Table 1, into a standard ICER calculation (shown in equation 11 using equations $8-11$. This can then be simplified to:

$$
I C E R=\frac{C_{S}}{(1-\beta) r E_{S}}
$$


Using these same variables and assumptions, the underlying net monetary benefit $(N M B)$ expression can be simplified to:

$$
N M B=\lambda(1-\beta) r E_{S}-C_{S}
$$

Where $\lambda$ is the ceiling ratio of willingness to pay for the health outcome. While ICER and net benefit calculations are not made in this way in practice, these expressions become useful in discussing the relationship between risk and cost-effectiveness. In equation 12, the denominator represents the expected incremental benefit of screening an individual of risk $r$. Here we assume a true positive screen $\left(E_{S}\right)$ to be beneficial (and of a value of 1) and fixed across risk levels. This means that the relationship between individual risk $(r)$ and the expected incremental benefit of screening is positive and linear. Similarly, if we assume that the cost of screening is positive and constant across risk levels, a higher level of risk (ceteris paribus) will be associated with a lower ICER. As such, the relationship between an individual's risk of developing a disease and the expected cost-effectiveness of screening them must be positive.

Adopting figures relating to existing screening programmes [13, 14, 15], shown in Table 2, we can estimate cost-effectiveness ratios for individuals at different levels of risk. In the diabetic retinopathy study, the authors estimate prevalence to be $14.1 \%$, but that only 4 in 5 people attend screening. For now we assume attendance to be $100 \%$. Figure 2 shows that the higher the level of individual risk $(r)$, the lower the resulting cost per positive screen. In this graph we can see that the cost per true positive screen is extremely sensitive to differences in individual risk when risk is very low. Figure

\begin{tabular}{l|l|l} 
& Diabetic retinopathy & Colorectal cancer \\
\hline$\alpha$ & 0.49 & 0.46 \\
$\beta$ & 0.02 & 0.02 \\
$C_{S}$ & $£ 21$ & $£ 9.20$ \\
$I C E R$ & $£ 209$ per true positive & $£ 5290$ per true positive \\
$E_{S}$ & 1 & 1
\end{tabular}

Table 2: Paramaters for diabetic retinopathy screening [13] and colorectal cancer screening[14, 15], compared with no screening 
2 only shows risk levels up to 0.1 and ICERs up to $£ 10,000$. The curve is almost Lshaped. This is primarily because we have not accounted for errors in the estimation of risk. Greater error in the risk engine, or a higher false negative rate, would cause the curve to flatten. This is because the rate of true positives at lower risk levels would be lower. The curve will also become flatter with a greater cost of screening, and become more L-shaped if an intervention is more effective. In this respect the shape of the curve is an indication of the cost-effectiveness of the intervention. Here we have assumed true positive screening outcomes to be of equal value for diabetic retinopathy and for colorectal cancer, so the cheaper intervention - for colorectal cancer - is more cost-effective.

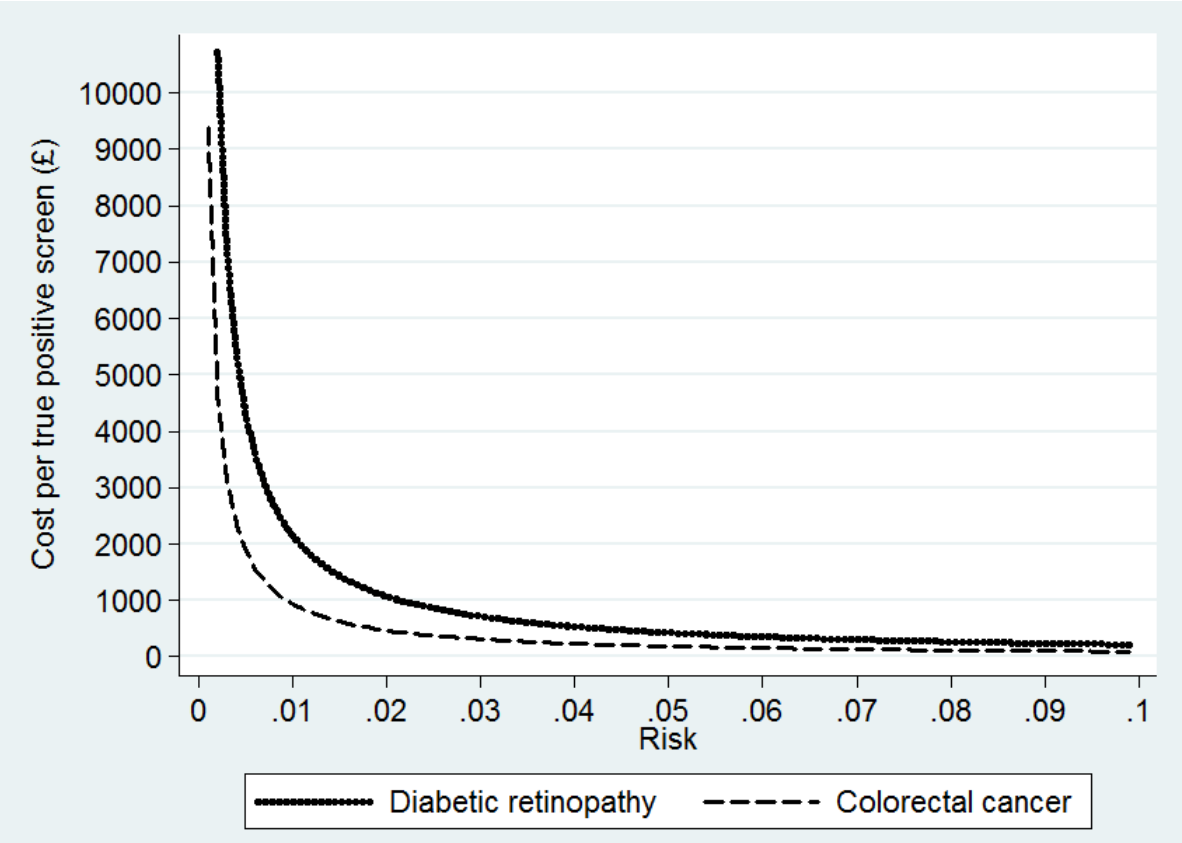

Figure 2: The relationship between risk and cost-per-outcome 


\subsection{Optimisation}

In addition to the screening procedure itself, screening interventions can be varied in two ways; the risk level at which an individual is screened and the frequency with which screening should take place. This dynamic applies to all screening programmes, but existing programmes tend to keep these two possible variants fixed. Time and risk are both continuous variables, infinitely divisible, meaning that we are presented with an infinite number of possible interventions ranging from constant screening for the whole asymptomatic population to no screening. For a given individual with a given level of risk, one (or more) of these infinite possibilities must represent the most costeffective intervention. At present there is not a generally accepted way of estimating this.

Figure 2 highlights that, for a given level of willingness to pay per expected gain, there will be a corresponding minimum level of risk at and above which it is costeffective to screen an individual, and below which the expenditure would not be deemed cost-effective. Current practice does not estimate this figure. This therefore demonstrates the potential for optimality in risk-based screening. For a given set of cost and outcome estimates, and for a given willingness to pay, there is likely to be a level of risk at which the net benefit of screening becomes positive.

We can use the net benefit approach to estimate whether, at a given level of willingness to pay per true positive screen, it is cost-effective to screen an individual with a given level of risk. Figure 3 shows the relationship between individual risk and net benefit. Here, for the purpose of the demonstration, we assume that the willingness to pay per true positive screen is $£ 500$ for diabetic retinopathy and $£ 1000$ for colorectal cancer. As can be seen in equation 13 , the slope is defined by the willingness to pay and the intercept, in our case, is defined by the cost. By solving for $r$ at our assumed $\lambda \mathrm{s}$, we obtain the minimum level of risk at which individuals should be screened. In the case of diabetic retinopathy, where $\lambda=£ 500$, the minimum level of individual risk is 0.0429 . In the case of colorectal cancer, where $\lambda=£ 1000$, the minimum level

of individual risk is 0.0094 . James et al find that, for systematic diabetic retinopathy screening, the incremental cost per true positive is $£ 209$ [13]. At a willingness to pay 
of $£ 209$, for example, it would be cost-effective to screen anybody with a risk level of 0.102 or higher. This assumes that the time period used in the estimation of the hazard rate matches the screening interval.

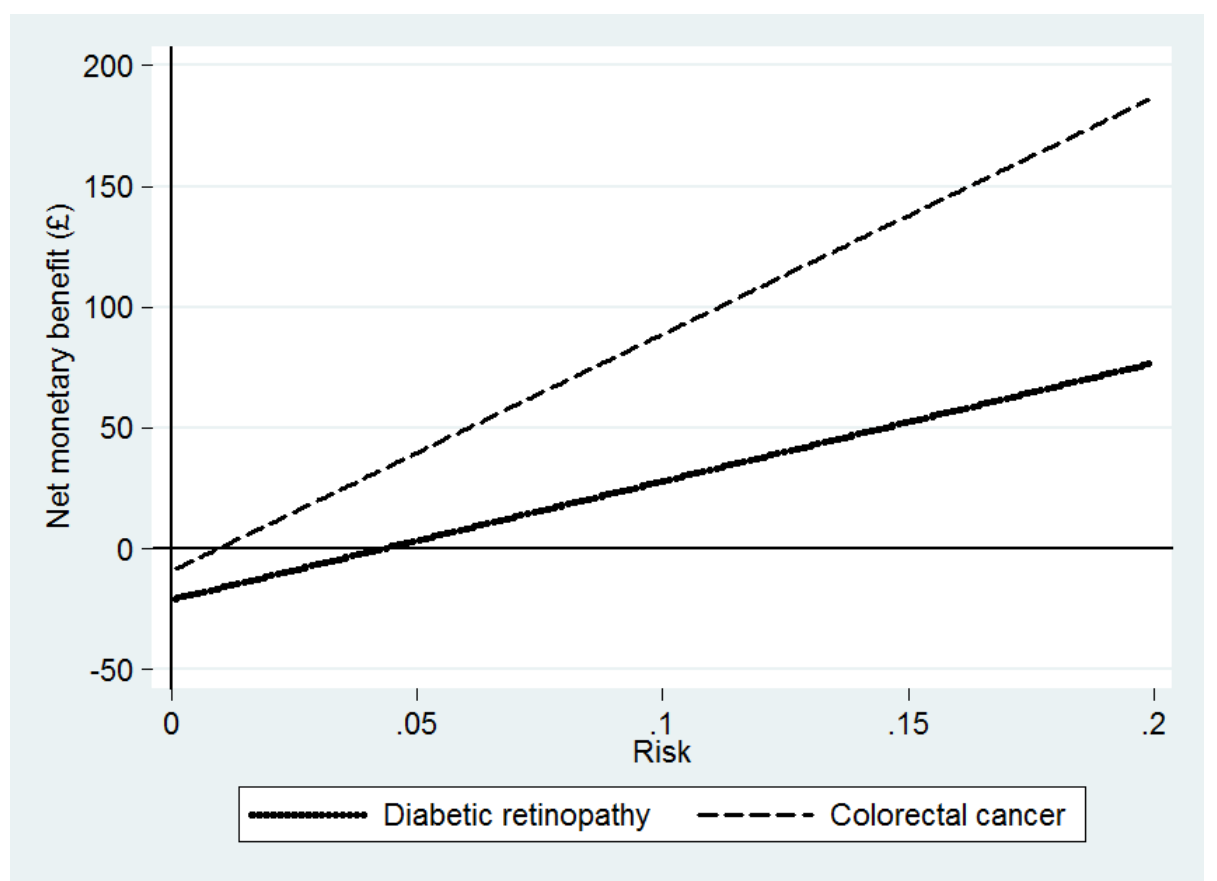

Figure 3: The relationship between risk and net benefit

These relationships are intuitive. The higher an individual's level of risk, the more likely it is that they will screen positive and therefore benefit as intended from the screening programme. This means that the expected cost per outcome for an individual with a high risk is low, while for those at a low risk it may be very high.

As explained above, screening interventions can also be varied in terms of the frequency of their administration. In practice, the question is not one of frequency, but one of recall period. An individual will only receive a follow-up screen if they are screened negative at their first screen. In an optimised programme, the time to next screen would be decided following each negative screening outcome, rather than a screening frequency being defined at an arbitrary time point. The incremental costeffectiveness of subsequent screens can be characterised in the same way as the first; 
the inputs may have changed, but the decision process is the same. As such, the relationship rationalises to that already discussed.

For some programmes it may be more useful to estimate an optimal recall period for an individual at a given point in time, rather than to define a minimum level of risk for population screening. Individual risk is usually estimated in relation to a clinically relevant period of time. For diabetic retinopathy, the risk estimation may be risk of developing sight-threatening retinopathy within 1 year. For colorectal cancer the risk may relate to 10 years or a lifetime. Diabetic retinopathy is one disease area in which screening is usually recurrent, and we use this example here to demonstrate the definition of an optimal recall period. In order to establish an optimal recall period, it is necessary to decide on the lowest practical screening interval. For example, a screening clinic may have the administrative capacity of recalling individuals on a daily, weekly, monthly or yearly basis. Maintaining the same assumptions as above, and assuming that individuals' hazard ratios are estimated based on a time-at-risk of 1 year and that screening clinics are capable of recalling individuals for screening on a weekly basis, we can estimate the recall time at which net monetary benefit becomes positive. This is simply a matter of estimating the cost-effectiveness of screening an individual at various periods of recall, accounting for the fact that risk increases in time. It is reasonable to presume that the higher an individual's level of risk, the more likely it is to be cost-effective to screen them within a shorter period of time. This is shown in Figure 4, in which individuals of annual risk 0.01, 0.02 and 0.03 are found to have optimal recall periods of 227, 113 and 75 weeks respectively. The optimal recall period, in terms of cost-effectiveness, can be found by solving for $N M B=0$ at any given level of individual risk. The relationship that this produces is shown in Figure 5 .

Decision modelling techniques are routinely used in the evaluation of screening programmes, and we believe it is these methods that offer the best opportunities for the incorporation of individual risk data. Modelling methods, and modern computer software, allow researchers to efficiently carry out thousands of simulations within short periods of time. Though it is not usually necessary, it is possible to evaluate an intervention hundreds of times with different hypothetical populations, so long as these populations are related in some consistent and quantifiable way. Therefore, a model 


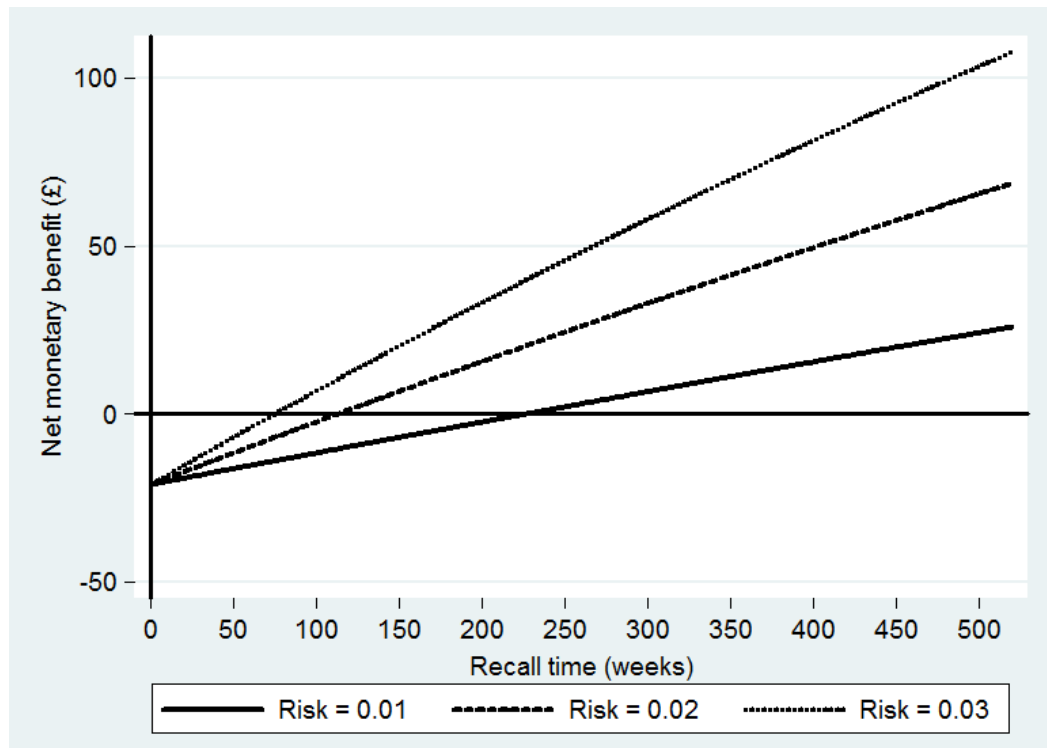

Figure 4: The relationship between recall period and net benefit

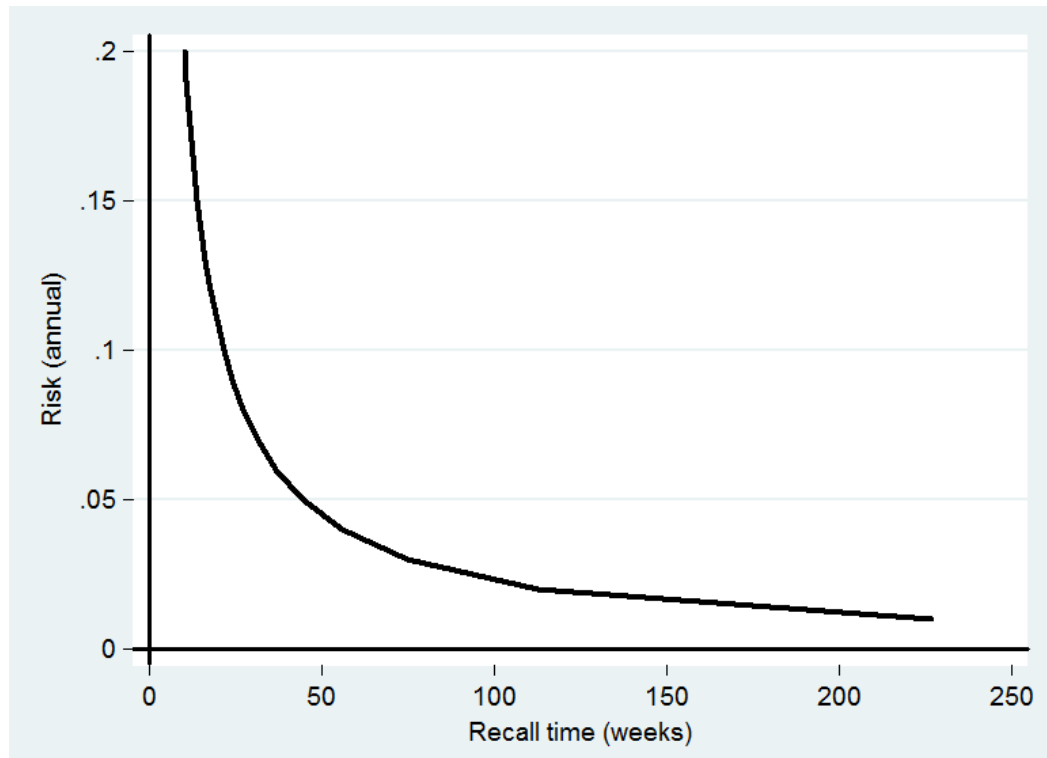

Figure 5: The relationship between risk and optimal recall period 
could be designed that evaluated a screening intervention for individuals of any given risk level. One could estimate whether or not screening was cost-effective, compared with no screening, for individuals at predicted risk levels from 0 to 1 . This would then enable a researcher to estimate the relationship between risk and cost-effectiveness and predict the risk level above which screening is cost-effective, and below which it is not. This, in turn, could inform the delivery of services. We seek to achieve this in future work for the case of screening for diabetic retinopathy.

\section{Discussion}

\subsection{Policy implications}

If, as we suggest, there is a quantifiable relationship between individual risk and costeffectiveness, it should arguably be represented and considered in economic evaluations of preventative interventions. As shown above, the optimally cost-effective screening interval for an individual is greater the lower their risk, because more regular screening is associated with higher costs and a lower average probability of a positive screen. This suggests that screening those with a lower risk less often could increase the costeffectiveness of their screening. This has been identified in a number of studies, as discussed above. In implementing a standardised programme it is very unlikely that screening will be optimal in terms of cost-effectiveness. The methods we propose would enable the identification of an optimal screening interval for an individual at any given risk level, resulting in the optimisation of a risk-based screening programme.

To fully optimise screening in the way described above, a programme of risk-based screening would need to be implemented. In practice, screening based on individual risk would require an extra level of intervention; a pre-screen. To some extent this stage already exists, in that individuals are identified as being a woman over 60 , for example, before being referred to screening. Risk-based screening would require a more comprehensive and detailed version of this. An individual's set of characteristics would need to be used to populate a risk algorithm in order to calculate a level of risk. This level of risk would then be used to define the appropriate screening intervention 
for this individual, based on cost-effectiveness and other factors. This process would

likely be computerised and could be completed by an individual, at home, or by a physician. If the relationship between an individual's level of risk and the expected cost-effectiveness of screening them can be estimated, then this process would enable screening to be optimised for each individual.

It's important to note that, so long as treatment is effective and the risk-engine is sufficiently accurate, a risk-based screening programme will always be cost-effective at some level of risk. However, it may be the case that the threshold level of risk identifies only a very small population. Therefore, it is likely to be important to take account of the fixed costs of implementing a screening programme and consider that marginal costs are likely to be decreasing. Such a programme would also require consideration of accessibility, inclusivity and the communication of risk to patients.

\subsection{Ethical issues}

To implement a programme such as that described above, one would need to estimate incremental costs and benefits at the individual level. This is not how economic evaluations are currently carried out. However, with the rise of personalised medicine, it seems likely that such economic evaluations may become necessary. The resulting curse of dimensionality will need to be confronted by economists and statisticians alike. Such an approach could become ethically problematic because evaluations may indicate that the most cost-effective regime is discriminatory. Efforts are underway to develop methods that systematically incorporate equity considerations into an economic evaluation. Once developed, such methods will be crucial in the evaluation of risk-based screening and other areas of personalised medicine. However, one must consider that existing screening programmes already discriminate based on age and gender. It may be that discriminating based on an estimated risk level is more favourable, as it only indirectly discriminates based on personal characteristics. 


\subsection{Limitations}

Clearly there are many assumptions in the framework described above, but we believe that most of these could be relaxed in a probabilistic model-based cost-effectiveness analysis. Our model assumes that costs, outcomes, sensitivity and specificity are all constant across risk. There are reasons to believe that all four of these parameters may differ across individuals of different risk levels. For example, individuals with a higher risk-level may have a more progressed disease and stand to gain less health from intervention. In our model, the relationship between risk and the expected benefit of the screening intervention is linear by definition. Similarly, a screening intervention may be less sensitive or specific for individuals with a lower risk level. Nevertheless, so long as these relationships can be estimated they can be built into a model accordingly. With sufficient observational data one could estimate risk-dependent costs, outcomes, sensitivities and specificities.

There are a number of important parameters that we have not included in our estimations, such as the disutility of screening, the rate of attendance and the predictive capability of the risk engine. The NSC states that screening should not cause harm. However, it is clear that a positive screen can lead to harm in the follow-up, as has been widely reported in relation to breast cancer screening [16]. This is not built into the model described above. Conversely there may be health benefits associated with a negative screening outcome. There may also be practical issues relating to screening partners or children. For example, screening a child, having already screened the mother, may be associated with a low marginal cost and a high expected benefit. By using a modelling approach in the way described above, important paramaters could be incorporated and the relationship with risk would be estimated in the same way.

\section{Conclusion}

In the future it is possible that screening will cease to be a one-size-fits-all type of intervention, and that risk-based screening programmes will be rolled-out. This is just part of a wider movement towards predictive and personalised medicine. Economists 
will need to be able to adapt their methods of evaluation to incorporate new types of information and interventions of a more variable nature. In this paper we have described the ways in which this might be done for screening. We have demonstrated that the cost-effectiveness of a screening intervention could potentially be optimised based on an individual's level of risk. and that methods of decision modelling are likely to enable this. Future work should attempt to observe this relationship and investigate the role of other variables such as attendance, sensitivity and specificity. 


\section{References}

[1] Dan YY, Chuah BYS, Koh DCS, Yeoh KG. Screening based on risk for colorectal cancer is the most cost-effective approach. Clinical gastroenterology and hepatology. 2012 Mar;10(3):266-71.e1-6. Available from: http://www.ncbi.nlm. nih.gov/pubmed/22100624.

[2] Lansdorp-Vogelaar I, van Ballegooijen M, Zauber AG, Boer R, Wilschut $\mathrm{J}$, Winawer SJ, et al. Individualizing colonoscopy screening by sex and race. Gastrointestinal endoscopy. 2009 Jul;70(1):96-108, 108.e1-24. Available from: http://www.pubmedcentral.nih.gov/articlerender.fcgi? artid=2805960\&tool=pmcentrez\&rendertype=abstract.

[3] Round JA, Jacklin P, Fraser RB, Hughes RG, Mugglestone Ma, Holt RIG. Screening for gestational diabetes mellitus: cost-utility of different screening strategies based on a woman's individual risk of disease. Diabetologia. 2011 Feb;54(2):256-63. Available from: http://www.ncbi.nlm.nih.gov/ pubmed/20809381.

[4] Aus G, Damber JE, Khatami A, Lilja H, Stranne J, Hugosson J. Individualized screening interval for prostate cancer based on prostate-specific antigen level: results of a prospective, randomized, population-based study. Archives of internal medicine. 2005;165(16):1857. Available from: http://archinte.ama-assn. org/cgi/content/abstract/165/16/1857

[5] Bradbury A, Olopade OI. The case for individualized screening recommendations for breast cancer. Journal of Clinical Oncology. 2006 Jul;24(21):3328-30. Available from: http://wWW.ncbi.nlm.nih.gov/pubmed/16801629.

[6] Brawley OW. Risk-based mammography screening: an effort to maximize the benefits and minimize the harms. Annals of internal medicine. 2012 May;156(9):662-3. Available from: http://wwW.annals.org/content/ 156/9/662. full 
[7] Scanlon PH. 10/66/01 - Development of a cost-effectiveness model for optimization of the screening interval in diabetic retinopathy screening. National Institute for Health Research Health Technology Assessment Programme; 2012. May. Available from: http://www.hta.ac.uk/project/2654

[8] Harding SP. RP-PG-1210-12016 - Introducing personalised risk based intervals in screening for diabetic retinopathy: development, implementation and assessment of safety, cost-effectiveness and patient experience. National Institute for Health Research Programme Grants for Applied Research; 2013. Available from: http: //www.ccf.nihr.ac.uk/PGfAR/about/Pages/Abstract.aspx?ID=7790

[9] Aspelund T, Thornórisdóttir O, Olafsdottir E, Gudmundsdottir a, Einarsdóttir $\mathrm{aB}$, Mehlsen $\mathrm{J}$, et al. Individual risk assessment and information technology to optimise screening frequency for diabetic retinopathy. Diabetologia. 2011 Oct;54(10):2525-32. Available from: http://wWw.ncbi.nlm.nih.gov/ pubmed/21792613.

[10] Mehlsen J, Erlandsen M, Poulsen PLg, Bek T. Individualized optimization of the screening interval for diabetic retinopathy: a new model. Acta ophthalmologica. 2010 Apr;p. 1-6. Available from: http://www.ncbi.nlm.nih.gov/pubmed/ 20384605

[11] Chalk D, Pitt M, Vaidya B, Stein K. Can the retinal screening interval be safely increased to 2 years for type 2 diabetic patients without retinopathy? Diabetes care. 2012 Aug;35(8):1663-8. Available from: http://www.ncbi.nlm.nih. gov/pubmed/22566535

[12] Davies R, Roderick P, Canning C, Brailsford S. The evaluation of screening policies for diabetic retinopathy using simulation. Diabetic Medicine. 2002 Sep;19(9):762-770. Available from: http: //wWW.ncbi.nlm.nih.gov/pubmed/12207814http://doi.wiley.com/ $10.1046 / \mathrm{j} .1464-5491.2002 .00773 . \mathrm{x}$

[13] James M, Turner DA, Broadbent DM, Vora J, Harding SP. Cost effectiveness analysis of screening for sight threatening diabetic 
eye disease. BMJ. 2000 Jun;320(7250):1627-31. Available from: http://wwW . pubmedcentral.nih.gov/articlerender.fcgi?artid= 27406\&tool=pmcentrez\&rendertype=abstract.

[14] Hardcastle JD, Chamberlain JO, Robinson MH, Moss SM, Amar SS, Balfour TW, et al. Randomised controlled trial of faecal-occult-blood screening for colorectal cancer. Lancet. 1996 Nov;348(9040):1472-1477. Available from: http://europepmc.org/abstract/MED/8942775.

[15] Whynes DK. Cost-effectiveness of screening for colorectal cancer: evidence from the Nottingham faecal occult blood trial. Journal of medical screening. 2004 Jan;11(1):11-5. Available from: http://www.ncbi.nlm.nih.gov/pubmed/ 15006108

[16] Independent UK Panel on Breast Cancer Screening. The benefits and harms of breast cancer screening: an independent review. The Lancet. 2012 Oct;380(9855):1778-1786. Available from: http://wwW.ncbi.nlm.nih. gov/pubmed/23117178http: //linkinghub.elsevier.com/retrieve/ pii/SO140673612616110. 